\title{
Enhancement of Dissolution Rate of Gliclazide Using Solid Dispersions: Characterization and Dissolution Rate Comparison
}

\author{
Sharmi Islam, Laboni Rani Dey, Mohammad Shahriar, Irin Dewan and S.M. Ashraful Islam \\ Department of Pharmacy, University of Asia Pacific, Dhaka, Bangladesh
}

\begin{abstract}
In this study solid dispersion (SDs) of gliclazide were prepared by solvent evaporation technique using poloxamer 407 as carrier. Drug carrier weight ratio were 1:1, 1:3 and 1:5. Physical mixtures of the same ratio were also prepared for comparison. The solid dispersions were investigated for drug loading and dissolution behavior and were found effective to enhance the solubility of gliclazide in dissolution medium significantly. Evaluation of the properties of the SDs was also performed by using Fourier-transform infrared (FTIR) spectroscopy and X-ray diffraction (XRD) studies. The FTIR spectroscopic studies showed the stability of gliclazide and absence of interaction between gliclazide and poloxomer 407. The XRD studies indicated the amorphous state of gliclazide in SDs. Dissolution data of SDs were compared by using both model dependant and model independent techniques. No significant difference in \% DE (dissolution efficiency) was found among the SDs. But the drug release rate from SDs differs from that of physical mixture. So, solid dispersion technique may be an effective way to enhance dissolution rate of gliclazide.
\end{abstract}

Key words: Gliclazide, solid dispersion, poorly water soluble, poloxamer 407

\section{Introduction}

Up to 40 percent of new chemical entities discovered by the pharmaceutical industries today are poorly soluble or lipophilic compounds that exhibit many difficulties in the development of pharmaceutical dosage forms due to their limited water solubility, slow dissolution rate and low bioavailability (Najmuddin et al., 2010). Now-a-days, pharmaceutical technology provides many approaches to enhance the dissolution rate of poorly soluble drugs. These include micronization, salt formation and formulation of the drug as a solid dispersion (SD). For many compounds, however, decreasing the particle size may not lead to a significant or adequate increase in bioavailability. Salt formation may also be problematic, particularly with neutral compounds and weak acids.

Solid dispersion technology is the science of dispersing one or more active ingredients in an inert matrix in the solid stage in order to achieve increased dissolution rate. Solid dispersions are prepared by various methods like fusion process, solvent process, fusion solvent process and supercritical fluid process (Sekiguchi and Obi, 1961). Solid dispersion method has been widely employed to improve the dissolution rate, solubility and oral absorption of poorly water soluble drugs. Numerous solid dispersion systems have been demonstrated in the pharmaceutical literature along with various hydrophilic carriers, such as polyethylene glycols, polyvinylpyrrolidone, hydroxypropyl methylcellulose, gums, sugar, mannitol and urea (Tanaka et al., 2006).

Chemically gliclazide is [1-(3-azabicyclo $(3,3,0)$ oct3-yl)-3-p-tolylsulfonylurea]. It is a second generation hypoglycemic sulfonylurea which is useful in the treatment of non-insulin dependent diabetes mellitus (NIDDM). Prior reports reveal that the drug shows good tolerability, low incidence of hypoglycemia, and a low rate secondary failure (Harrower, 1994). In addition, it has a potential for slowing the progression of diabetic retinopathy. For the reasons stated gliclazide appears to be a drug of choice in long term sulfonylurea therapy for the control of NIDDM (Harrower, 1994). Gliclazide is a white crystalline powder, relatively insoluble in water. The $\mathrm{pKa}$ of gliclazide is 5.8. It exhibits slow GI absorption rate and inter individual variations of its bioavailability (Palmer and Brogden, 1993). The slow absorption rate of drug usually originates from either poor dissolution of drug from the formulation or poor permeability of drug across

Correspondence to: S.M. Ashraful Islam, Department of Pharmacy, University of Asia Pacific, Dhaka, Bangladesh; Tel:+880-2-9664953, Ext-136; E-mail address: ashraf@uap-bd.edu 
GI membrane. The slow dissolution can be attributed, at least in part, to hydrophobicity of gliclazide powder as evidenced by poor wetting of powder surface by water. For poorly water soluble and highly permeable (class-II) drugs, the rate of oral absorption is often controlled by the dissolution rate in the gastrointestinal tract. Therefore, together with permeability, the solubility and or dissolution rate of a drug are key determinants of its oral bioavailability.

The objective of this work was to investigate the improvement in the solubility and dissolution rate of gliclazide by preparing solid dispersion with poloxamer 407. Poloxamer 407 facilitates solubilisation of poorly water soluble molecules like indomethacin (Dimitrova et al., 2000) or insulin (Barichello et al, 1999). Solubility of piroxicam in water was increased by 11 -fold by adding 22.5\% w/w Poloxamer 407 (Shin and Cho, 1997). Incorporation of Poloxamer 407 in solid dispersion of poorly water-soluble molecules, like nifedipine or piroxicam, led to marked improvement and thus promoted faster and more complete dissolution (Shin and Cho, 1997).

Dissolution enhancement of gliclazide by using PEG 6000 was also reported earlier (Biswal et al., 2008), but enhancement by using Poloxamer 407 was not reported earlier. So we used this in the current study to enhance the dissolution rate of this poorly water soluble drug.

Dissolution data were compared by using both model dependant (Zero order model, First order, Hixson-Crowell cube root law and Higuchi square root law) and model independent (difference factor (f1), similarity factor (f2), dissolution efficiency (\%DE) (Costa and Lobo, 2001) for comparison.

\section{Materials and Methods}

Materials: Standard of gliclazide was a kind gift from General Pharmaceuticals Ltd, Bangladesh. Poloxomer 407 was procured from BASF, Germany. All other chemicals and reagents used in this study were of analytical grade.

Preparation of solid dispersion: Solid Dispersions of gliclazide in poloxomer 407 in different weight ratios (1:1, $1: 3,1: 5)$ were prepared by solvent evaporation method and denoted as SD 1/1, 1/3 and 1/5 respectively. Gliclazide was dissolved in sufficient amount of methanol and the carrier was added. The solvent was then completely evaporated at $40-45^{\circ} \mathrm{C}$ and the resulting residue was dried under vacuum for $3 \mathrm{~h}$, stored in desiccators at least overnight, ground in a mortar, and passed through a \#100 sieve.

Preparation of physical mixer of gliclazide: Gliclazide and polymer (poloxamer 407) at different ratio (1:1, 1:3 and 1:5) were weighed by a calibrated balance (AY-200, Shimadzu, Japan) and physical mixtures were prepared by light trituration for 2 minutes by using mortar pestle. The mixture was passed through a 40 mesh size sieve. The prepared mixture was then filled in glass vials and coded as PM1/1, PM $1 / 3$ and PM1/5, sealed and stored in a desiccator till further use.

Determination of drug content in solid dispersions (SDs) and physical mixtures (PMs): To determine the potency, $\mathrm{SD}$ and $\mathrm{PM}$ equivalent to $10 \mathrm{mg}$ gliclazide was taken and dissolved in $100 \mathrm{ml}$ phosphate buffer $\mathrm{pH} 7.4$, described as dissolution medium, in BP-2010 for gliclazide tablet. Then the solution was filtered and assayed by Shimadzu UV/Visible double beam spectrophotometer. Absorbance of the above solution was measured at $226 \mathrm{~nm}$ and $290 \mathrm{~nm}$ using phosphate buffer $\mathrm{pH}$ 7.4 as blank. Absorbance was calculated by subtracting the absorbance at $290 \mathrm{~nm}$ from the absorbance at $226 \mathrm{~nm}$ as described in BP 2010. Finally the amount of drug in each formulation was calculated.

\section{Solid State Characterization of SDs}

Fourier-transform infrared spectroscopy: Fouriertransform infrared (FT-IR) spectra were obtained by using an FT-IR spectrometer-430. The samples (gliclazide or SDs) were previously ground and mixed thoroughly with potassium bromide, an infrared transparent matrix, at 1:5 (Sample: $\mathrm{KBr}$ ) ratio, respectively. The $\mathrm{KBr}$ discs were prepared by compressing the powder at a pressure of 5 tons for $5 \mathrm{~min}$ in a hydraulic press. Scans were obtained at a resolution of $4 \mathrm{~cm}^{-1}$, from 4000 to $500 \mathrm{~cm}^{-1}$.

$X$-ray diffraction: X-ray powder diffraction patterns were obtained at room temperature using a $\mathrm{D} \quad 8$ ADVANCE X-ray diffractometer (BRUKER, Germany) with $\mathrm{Cu}$ as anode material and graphite monochromator, operated at a voltage of $35 \mathrm{kV}$ and $20 \mathrm{~mA}$ current. The samples were analysed in the $2 \theta$ angle range of $5^{\circ}-70^{\circ}$ and the process parameters were set as: scan step size of $0.02^{\circ}(2 \theta)$, and scan step time of 0.5 degree $/ \mathrm{min}$.

In vitro dissolution study: Dissolution method described in BP 2010 was used to determine the percent 
drug release. The tests were performed for the pure gliclazide, physical mixture and solid dispersions, using dissolution test apparatus type II (Veego, VDA-8DR, USP Standards) using $900 \mathrm{ml}$ of phosphate buffer PH 7.4 as dissolution medium. The temperature of the medium was maintained at $37^{\circ} \mathrm{C} \pm 0.5^{\circ} \mathrm{C}$ throughout the experiment. The samples containing $80 \mathrm{mg}$ of gliclazide or its equivalent solid dispersions and physical mixture were placed in the dissolution medium. Paddle was used at a stirring rate of $100 \mathrm{rpm}$. A $10 \mathrm{ml}$ aliquot was withdrawn at predetermined time intervals of at 2, 5, 15,30, 40 and 60 minutes and then $10 \mathrm{ml}$ of fresh dissolution medium was replaced to maintain the constant volume of dissolution medium. From the samples collected, $1 \mathrm{ml}$ was diluted with dissolution medium and the absorbance of the diluted solutions were measured at $226 \mathrm{~nm}$ and $290 \mathrm{~nm}$ using Shimadzu UV/Visible double beam spectrophotometer (Shimadzu, Japan) against dissolution medium as blank. Percentage of drug release was calculated using the equation obtained from the standard curve prepared in the media.

\section{Comparison of Dissolution Data by Model Dependent Methods}

To study the release kinetics, data obtained form in vitro drug release study were tested with the following mathematical model.

Zero order equation: The equation assumes that the cumulative amount of drug release is directly related to time. The equation may be as follows:

$C=K_{0} t$

Where, $K_{0}$ is the zero order rate constant expressed in unit concentration/time and $t$ is time. A graph of concentration vs time would yield a straight line with a slope equal to $K_{0}$ and intercept the origin of the axes.

First order equation: The release behavior of first order equation is expressed as log cumulative percentage of drug remaining vs time. The equation may be as follows (Wagner, 1969)

$\log C=\log C_{0}-k t / 2.303$

Where,

$C=$ The amount of drug un-dissolved at $\mathrm{t}$ time,

$C_{0}=$ Drug concentration at $t=0$,

$k=$ Corresponding release rate constant.

Higuchi square root law: The Higuchi release model describes the cumulative percentage of drug release vs square root of time. The equation may be as follows (Higuchi, 1961):

$Q=K \sqrt{ } t$

Where, $Q=$ the amount of drug dissolved at time $t . K$ is the constant reflecting the design variables of the system. Hence, drug release rate is proportional to the reciprocal of the square root of time.

Hixson-Crowell cube root law: It is the law that represents idea about the evaluation of drug release pattern changes with the surface area and the diameter of the particles. It is mentioned as the cube root of the percentage of drug remaining in the matrix vs time (Hixon and Crowell, 1931). The equation is as follows

$Q_{0}{ }^{1 / 3}-Q_{t}^{1 / 3}=k_{H C} \times t$

Where, $Q_{0}=$ Initial amount of the drug in the tablets; $Q_{t}=$ The amount of drug release in time $\mathrm{t} ; k_{H C}=$ The rate constant for the Hixson-Crowell cube root law

\section{Comparison of Dissolution Data by Model Independent Methods}

Data obtained form in vitro drug release studies were tested with the different model independent technique: dissolution efficiency (\%DE) difference factor (f1), similarity factor (f2).

Dissolution efficiency (\%DE) was employed to compare the drug release from different solid dispersion. Dissolution efficiency is the area under the dissolution curve within a time range $\left(t_{1}-t_{2}\right)$ expressed as a percentage of the dissolution curve at maximum dissolution, over the same time frame (Anderson et al., 1998). This was calculated from the equation:

$$
D E=\frac{\int_{t_{1}}^{t_{2}} y \cdot d t}{y_{100} \times\left(t_{2}-t_{1}\right)} \times 100
$$

where, $y$ is the percentage dissolved at time $t$

Difference factor $\left(f_{1}\right)$ and similarity factor $\left(f_{2}\right)$ were calculated to find out similarity of solid dispersion and physical mixture. Difference factor $f_{1}$ is the percentage difference between two curves at each point and is a measurement of the relative error between the two curves. The similarity factor $\left(f_{2}\right)$ is a logarithmic reciprocal square root transformation of the sum of squared error and is a measurement of the similarity in the percent (\%) dissolution between the two curves. The following 
equations were used to calculate difference factor $f_{1}$ and similarity factor $\mathrm{f}_{2}$ :

$$
\begin{aligned}
& \mathrm{f}_{1}=\left\{\frac{\sum_{\mathrm{t}=1}^{\mathrm{n}}\left|\mathrm{R}_{\mathrm{t}}-\mathrm{T}_{\mathrm{t}}\right|}{\sum_{\mathrm{t}=1}^{\mathrm{n}} \mathrm{R}_{\mathrm{t}}}\right\} \mathrm{X} 100 \\
& \mathrm{f}_{2}=50 \log \left\{\left(1+\frac{1}{\mathrm{n}} \sum_{\mathrm{i}=1}^{\mathrm{n}}\left(\mathrm{R}_{\mathrm{t}}-\mathrm{T}_{\mathrm{t}}\right)^{2}\right)^{-0.5} \mathrm{X} 100\right\}
\end{aligned}
$$

where, $\mathrm{n}$ is the number of time points, $\mathrm{Rt}$ is the dissolution value of reference product at time $t$ and $T_{t}$ is the dissolution value for the test product at time $t$.

Similarity factor $f_{2}$ has been adopted by FDA (1997) and the European Agency for the Evaluation of Medicinal Products (EMEA, 2001) by the Committee for Proprietary Medicinal Products (CPMP) to compare dissolution profile. Two dissolution profiles are considered similar and bioequivalent, if $f_{1}$ is between 0 and 15 and $f_{2}$ is between 50 and 100 (FDA, 1997).

\section{Results and Discussion}

Physical appearance and potency of prepared solid dispersion: The gliclazide solid dispersions were prepared employing solvent evaporation method. All solid dispersions were white fine powders. No discoloration was observed during preparation of SD. Prior to in-vitro dissolution study the prepared solid dispersion was subjected to potency test. Three measurements were performed and Potency \pm SD of different SD and PM with pure drug is shown in table1. Potency of gliclazide was between $95-101 \%$.
Table 1. Potency of pure drug, prepared SDs and PMs.

\begin{tabular}{ccc}
\hline Sl. No. & Item & $\%$ Potency \pm SD $(\mathrm{n}=3)$ \\
\hline 1 & Pure Gliclazide & $99.38 \pm 0.55$ \\
2 & SD 1/1 & $100.77 \pm 0.78$ \\
3 & SD 1/3 & $96.65 \pm 0.72$ \\
4 & SD 1/5 & $98.18 \pm 0.71$ \\
5 & PM 1/1 & $97.42 \pm 0.82$ \\
6 & PM 1/3 & $99.46 \pm 0.82$ \\
7 & PM 1/5 & $99.25 \pm 0.92$ \\
\hline
\end{tabular}

\section{Solid State Characterization}

The solid state characterization of drug and SD were investigated using FTIR and XRD to find out chemical interaction and crystalline nature of gliclazide and SD.

Fourier-transform infrared spectroscopy: Fouriertransform infrared (FT-IR) spectroscopy was used to characterize possible interactions between the drug and the carrier in solid state. The IR spectra of SDs and PMs were compared with the standard spectrum of gliclazide. The IR spectrum of gliclazide was characterized by the absorption of carbonyl $(\mathrm{C}=\mathrm{O})$ and $\mathrm{NH}$ group at $1708.96 \mathrm{~cm}^{-1}$ and $3273.26 \mathrm{~cm}^{-1}$, respectively. In the spectra of SDs and PMs, peak for the carbonyl absorption of $(\mathrm{C}=\mathrm{O})$ was in the same frequencies $\left(1708.96 \mathrm{~cm}^{-1}\right)$ but the peak for $\mathrm{NH}$ group shifted to 3274.22 SDs. So we can say that, there is no drug carrier interaction in SD and PM. IR spectra of Gliclazide, polymer and each sample of SD are shown in figure 1-3.

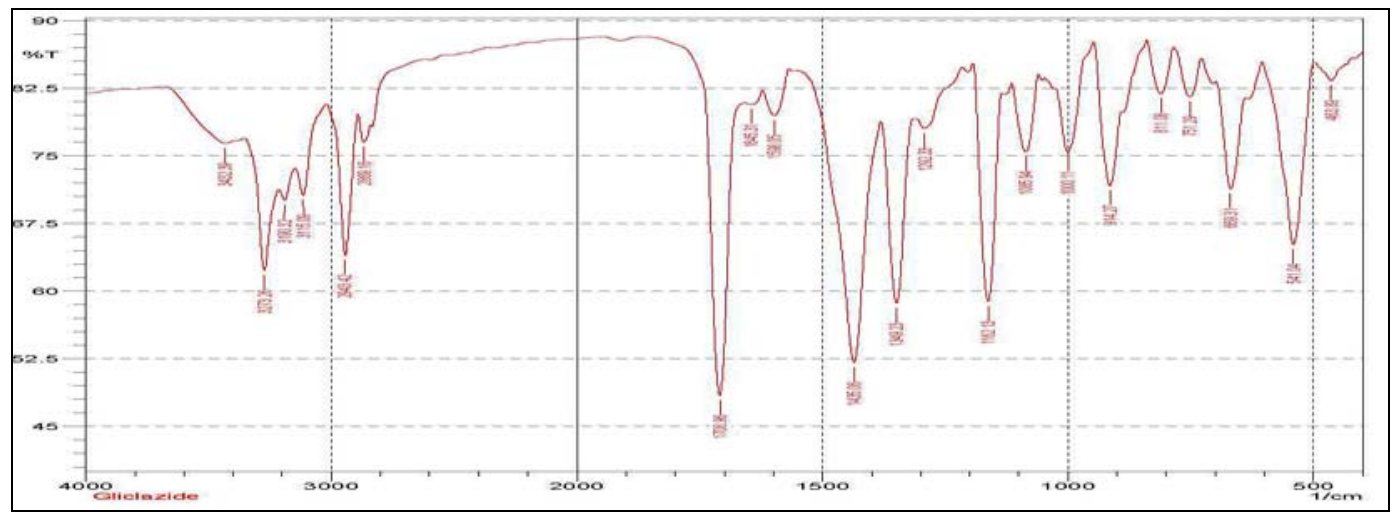

Figure 1. FT-IR spectrum of gliclazide. 


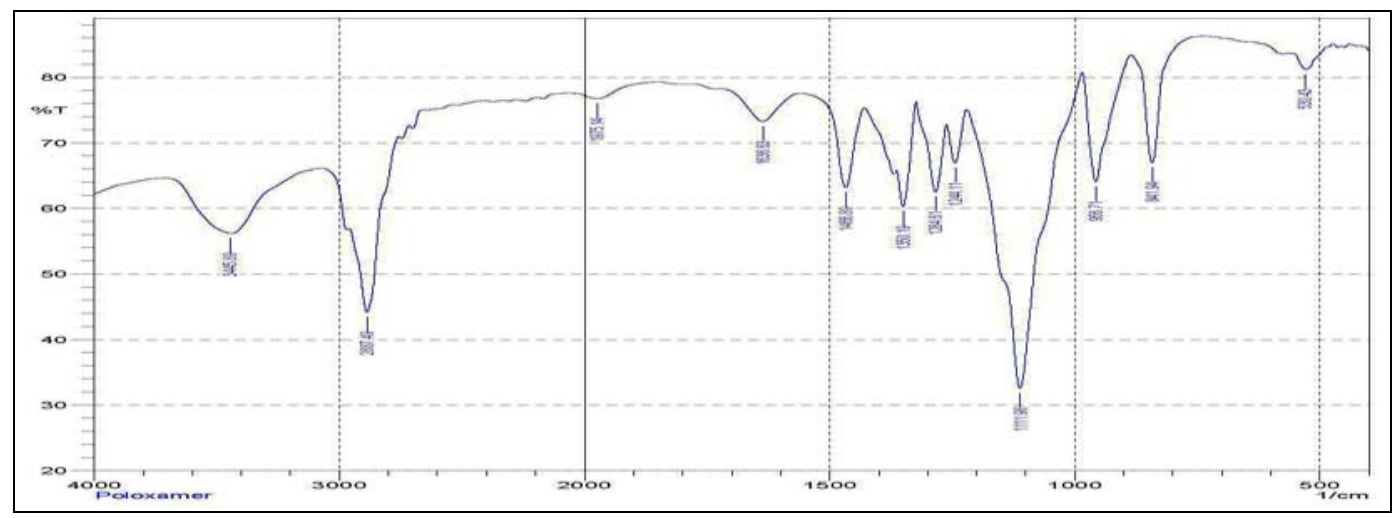

Figure 2. IR spectrum of poloxamer 407.

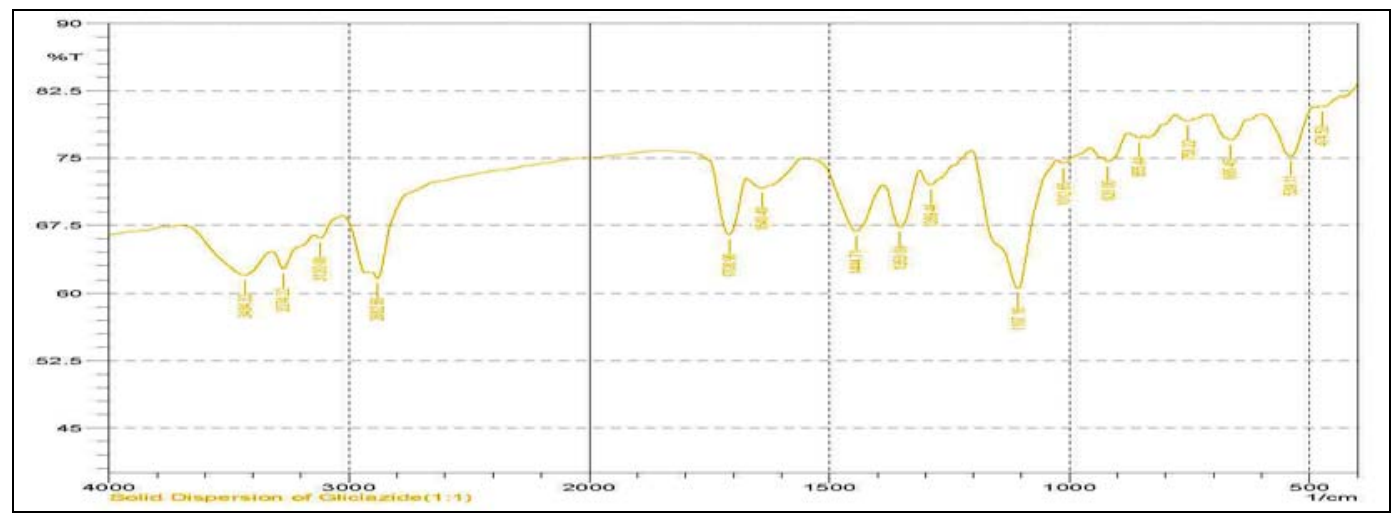

Figure 3. FT-IR spectrum of solid dispersion of gliclazide (SD1/1).

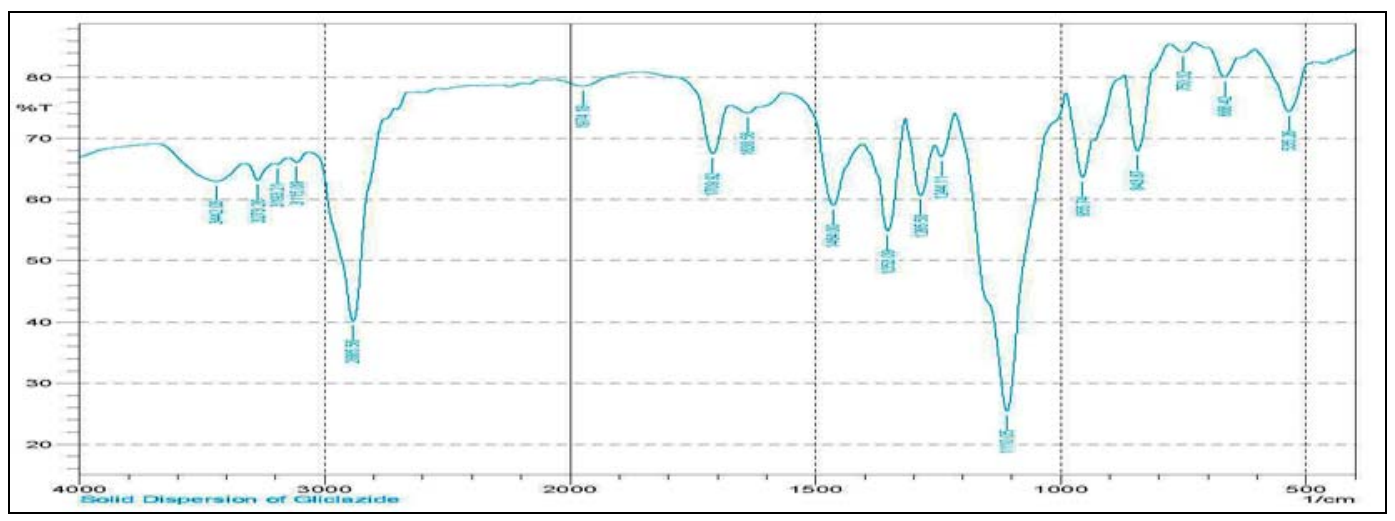

Figure 4. FT-IR spectrum of solid dispersion of gliclazide ( SD1/5).

$X R D$ study to characterize the SDs: Solid state characterization of drug and SD were investigated using XRD to find out crystalline nature of gliclazide and solid dispersion (SD 1/5). The diffraction spectrum of pure gliclazide showed that the drug was crystalline in nature as it was emonstrated by numerous peaks. Numerous diffraction peaks of gliclazide were observed at 20 of 10.48, 18.1620 .78 and 22.2 (Figure 5) indicating crystalline Gliclazide. Some changes in the peak positions of Gliclazide were observed in SDs $1 / 5$. The prominent peaks in the SD were 19.08 and 23.28 Peak intensity was also decreased in SD. 
The relative reduction of diffraction intensity of gliclazide in SD preparations at these angles suggests that the size of the crystals was reduced. The results of this study imply that gliclazide is present in partially amorphous or microcrystalline form in the SDs. X-ray diffraction spectrum of pure gliclazide and SD1/5 are shown in fig: 56.

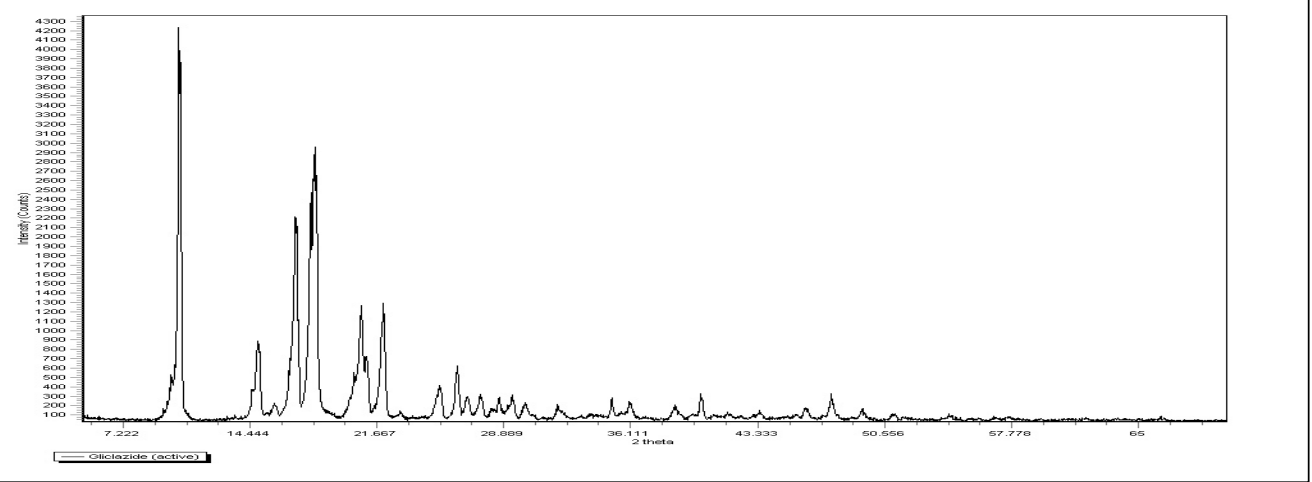

Figure 5. X-ray diffraction (XRD) patterns of pure gliclazide.

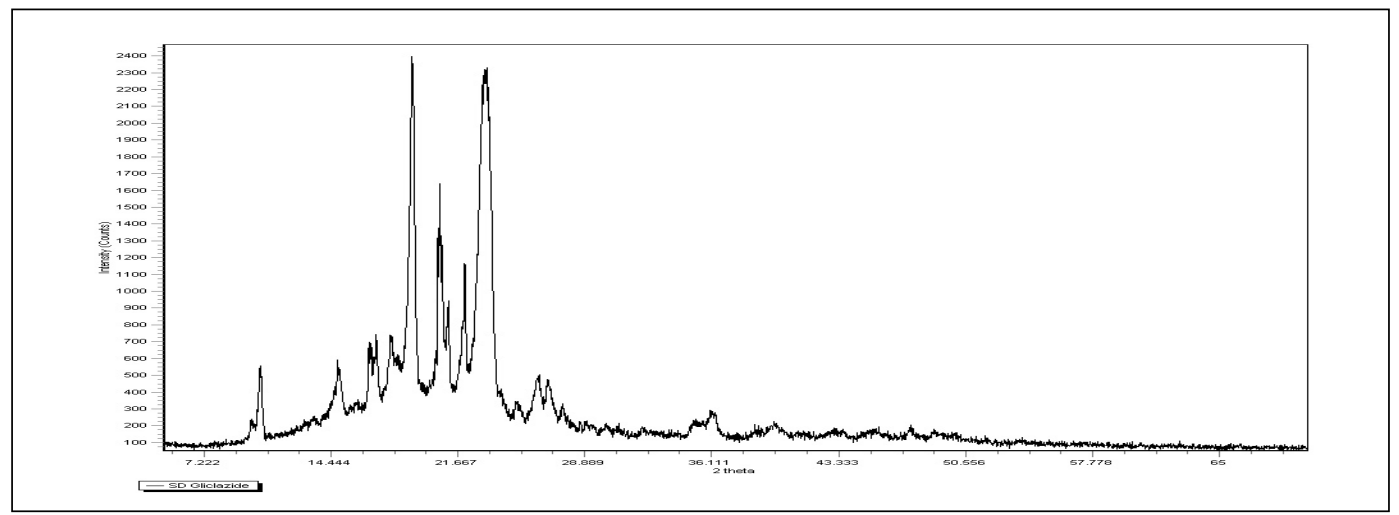

Figure 6. X-ray diffraction (XRD) patterns of SD 1/5 of gliclazide.

\section{Dissolution profile of Gliclazide from binary Solid Dispersion}

The purpose of the study was to increase the dissolution rate of gliclazide, a poorly water soluble drug. Poloxamer 407 in different ratio was used to prepare SDs and PMs for this purpose. All solid dispersions prepared by solvent evaporation method were found to be granular, fine and free flowing.

Here $80 \mathrm{mg}$ of pure gliclazide powder was used for dissolution study. It was found only $4.8 \%$ was released after 5 minutes, $49.3 \%$ was released after 40 minutes and $65 \%$ was released after one hour.

Mixture of gliclazide and poloxamer 407 (PM 1/1, PM 1/3 and PM 1/5) were used for dissolution study. It was found that $42.2 \%$ for PM $1 / 1$ and $45.2 \%$ for PM $1 / 5$ were release after 5 minutes. $75 \%$ for PM $1 / 1$ and $85 \%$ for PM 1/5 were released after 40 minutes (Figure 7).

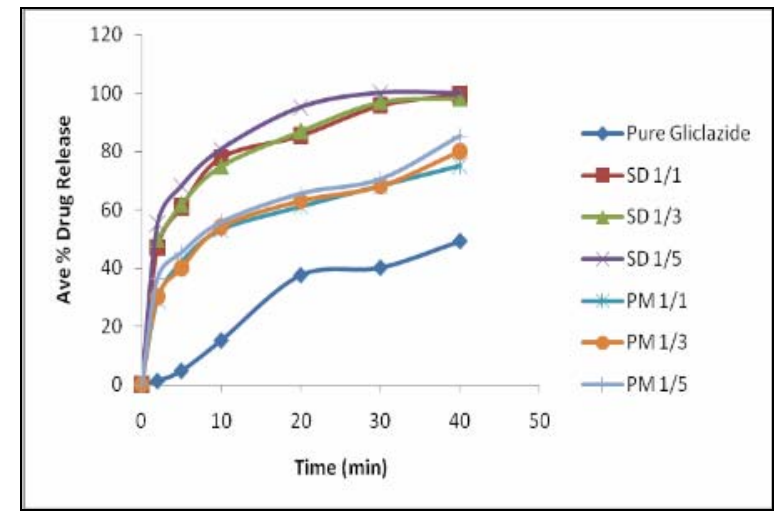

Figure 7. Percent release of active drug (gliclazide), solid dispersion (SD1/1, SD1/5) and physical mixture (PM 1/1, PM $1 / 5)$.

Solid dispersion of gliclazide and Poloxamer 407 (SD 1/1, SD 1/3 and SD 1/5) were used for dissolution study. 
Drug released from SD1/1 was found $60 \%$ within 5 minutes, $99.55 \%$ within 40 minutes and drug release from SD1/5 was found $68 \%$ within 5 minutes and $100 \%$ within 30 minutes.

All the solid dispersions show better release profiles compared to the physical mixtures and the drug itself. All solid dispersions show 100\% release within 30 minutes where the drug and physical mixtures show delayed release $(100 \%$ release at 45 or 60 minutes). We also observed that drug release rate was increased with increasing the water soluble polymer (poloxamer 407).

In solid dispersion a fraction of the drug might molecularly disperse in the matrix. When the solid dispersion is exposed to aqueous media, the carrier dissolves and the drug releases as fine colloidal particles. But in case of physical mixer there drugs are not molecularly dispersed in the matrix. For this reason drug release from solid dispersion is more than physical mixer.

So, from the present released study we can say that solid dispersion may be an effective method to enhance dissolution of gliclazide.

\section{Drug Release Kinetics}

In this study SDs were prepared by solvent evaporation method. Drug released from SDs and PMs were analyzed by Zero order model, First order, HixsonCrowell cube root law and Higuchi square root equation. $\mathrm{Y}$-equation $(\mathrm{Y}=\mathrm{aX}+\mathrm{b})$ and correlation co-efficient $\left(\mathrm{R}^{2}\right)$ of solid dispersion and physical mixture are shown in table 2. The data shows that only pure drug follows $1^{\text {st }}$ order, Higuchi release model but in case of SDs, $\mathrm{R}^{2}$ values were less than 0.693 (Table 2). This may be due to the slow release rate of pure drug and higher release rate of SDs. SDs and PMs followed $1^{\text {st }}$ order release kinetics.

Table 2. Drug release kinetics for active drug (gliclazide), solid dispersion (SDs) and physical mixture (PMs).

\begin{tabular}{ccccccccc}
\hline \multirow{2}{*}{ tem } & \multicolumn{2}{c}{ Zero Order } & \multicolumn{2}{c}{$1^{\text {st }}$ Order } & \multicolumn{2}{c}{ Higuchi Model } & \multicolumn{2}{c}{ Hixson-Crowell Model } \\
\cline { 2 - 8 } & Y equation & $\mathrm{R}^{2}$ & Y equation & $\mathrm{R}^{2}$ & Y equation & $\mathrm{R}^{2}$ & \multicolumn{2}{c}{ Y equation } \\
\hline Gliclazide & $\mathrm{y}=1.320 \mathrm{x}+1.020$ & 0.945 & $\mathrm{y}=-0.007 \mathrm{x}+2.001$ & 0.963 & $\mathrm{y}=8.794 \mathrm{x}-7.795$ & 0.928 & $\mathrm{y}=0.080 \mathrm{x}+1.015$ & 0.789 \\
SD 1/1 & $\mathrm{y}=1.872 \mathrm{x}+38.06$ & 0.671 & $\mathrm{y}=-0.051 \mathrm{x}+1.934$ & 0.947 & $\mathrm{y}=14.46 \mathrm{x}+18.97$ & 0.888 & $\mathrm{y}=0.065 \mathrm{x}+2.628$ & 0.372 \\
SD 1/3 & $\mathrm{y}=1.842 \mathrm{x}+38.87$ & 0.662 & $\mathrm{y}=-0.042 \mathrm{x}+1.857$ & 0.976 & $\mathrm{y}=14.27 \mathrm{x}+19.94$ & 0.88 & $\mathrm{y}=0.065 \mathrm{x}+2.649$ & 0.364 \\
SD 1/5 & $\mathrm{y}=1.813 \mathrm{x}+43.58$ & 0.599 & $\mathrm{y}=-0.067 \mathrm{x}+1.916$ & 0.989 & $\mathrm{y}=14.40 \mathrm{x}+23.79$ & 0.837 & $\mathrm{y}=0.063 \mathrm{x}+2.746$ & 0.339 \\
PM 1/1 & $\mathrm{y}=1.472 \mathrm{x}+24.54$ & 0.746 & $\mathrm{y}=-0.013 \mathrm{x}+1.879$ & 0.9 & $\mathrm{y}=11.06 \mathrm{x}+10.54$ & 0.934 & $\mathrm{y}=0.062 \mathrm{x}+2.275$ & 0.412 \\
PM 1/3 & $\mathrm{y}=1.564 \mathrm{x}+23.95$ & 0.778 & $\mathrm{y}=-0.014 \mathrm{x}+1.890$ & 0.925 & $\mathrm{y}=11.59 \mathrm{x}+9.602$ & 0.948 & $\mathrm{y}=0.063 \mathrm{x}+2.264$ & 0.428 \\
PM 1/5 & $\mathrm{y}=1.583 \mathrm{x}+26.99$ & 0.755 & $\mathrm{y}=-0.016 \mathrm{x}+1.879$ & 0.919 & $\mathrm{y}=11.79 \mathrm{x}+12.31$ & 0.928 & $\mathrm{y}=0.062 \mathrm{x}+2.361$ & 0.403 \\
\hline
\end{tabular}

\section{Model Independent Analysis of Dissolution Data}

The $\%$ DE indicates the overall performance of the carrier in drug release. \% DE of all the prepared SDs along with pure drug and PMs were calculated $(n=6)$ and shown in table 3 . The results indicate that solid dispersion is more effective to increase the dissolution rate than physical mixture as \% DE is more than $80 \%$ in case of SDs and around $65 \%$ in case of PMs. \% DE of pure drug is very low $(30.53 \%)$. \% DE increases with increase of carrier both in SDs and PMs. But the increase of \% DE is not proportional to the amount of carrier.
Table 3. Dissolution efficiencies (\%DE) of SDs and PMs.

\begin{tabular}{cccc}
\hline Item & $\mathrm{f}_{2}$ & $\mathrm{f}_{1}$ & \%DE \\
\hline SD 1/1 & 100.00 & 0.00 & 84.47 \\
SD 1/3 & 82.51 & 2.36 & 84.65 \\
SD 1/5 & 59.81 & 6.92 & 89.77 \\
PM 1/1 & 31.74 & 29.45 & 60.10 \\
PM 1/3 & 32.63 & 28.24 & 61.05 \\
PM 1/5 & 36.32 & 23.22 & 64.15 \\
Gliclazide & 13.63 & 68.20 & 30.53 \\
\hline
\end{tabular}

The percent drug release was also compared by difference factor $\left(f_{1}\right)$ and similarity factor $\left(f_{2}\right)$. SD 1/1 was used as reference to calculate the $f_{1}$ and $f_{2}$. Table 3 shows the $f_{1}, f_{2}$ values of different solid dispersion and physical mixture in respect of SD $1 / 5$ as a reference. Physical mixtures were more found different than solid dispersion as $\mathrm{f}_{2}$ is less. On the other hand, all the solid dispersions are 
found similar $\left(\mathrm{f}_{2}\right.$ is greater than 50$)$. So we can conclude that, although \% DE is different for different SDs but the difference in $\% \mathrm{DE}$ is not significant.

Again, $f_{1}, f_{2}$ values of different PMs in respect of PM $1 / 1$ were also calculated and shown in table 4 . All the PMs were found similar ( $f_{2}$ is greater than 50 ).

Table 4. Calculated difference factor $\left(f_{1}\right)$ and similarity factor $\left(f_{2}\right)$ of PMs.

\begin{tabular}{cccc}
\hline Item & $\mathrm{f}_{2}$ & $\mathrm{f}_{1}$ & $\% \mathrm{DE}$ \\
\hline PM 1/1 & 100.00 & 0.00 & 60.10 \\
PM 1/3 & 79.83 & 3.27 & 61.05 \\
PM 1/5 & 62.07 & 8.83 & 64.15 \\
Gliclazide & 25.66 & 54.92 & 30.53 \\
\hline
\end{tabular}

\section{Conclusion}

Solid dispersions of gliclazide with poloxamer 407 in different ratios were prepared to improve dissolution characteristics. Solvent evaporation method was employed to prepare solid dispersions. In vitro dissolution studies showed that solid dispersions were effective in increasing the dissolution of gliclazide and gave greater release rate than pure drug. Dissolution data were analyzed by model dependant and independent technique. The experiment proves that SDs of poloxomer 407 were better than PMs for higher drug release. So, Solid dispersion technique may be an effective technique to enhance dissolution rate of gliclazide. However, in vivo study is required for final selection of carrier.

\section{Acknowledgements}

The authors are thankful to University of Asia Pacific for supports and co-operations. We are also thankful to BCSIR, Bangladesh for XRD analysis and Center for Advanced Research in Sciences (CARS), Dhaka University, Bangladesh for FTIR analysis.

\section{References}

Anderson, N.H., Bauer, M., Boussac, N., Khan-Malek, R., Munden, P. and Sardaro, M. 1998. An evaluation of fit factors and dissolution efficiency for the comparison of in vitro dissolution profiles. J. Pharm. Biomed. Anal. 17, 811822.
Barichello, J. M., Morishita, M. , Takayama, K., Chiba, Y., Tokiwa, S. and Nagai. T. 1999. Enhanced rectal absorption of insulinloaded Pluronic F-127 gels containing unsaturated fatty acids. Int. J. Pharm. 183, 125-132.

Biswal, S., Sahoo, J., Murthy, P. N., Giradkar, R. P. and Avari J. G. 2008. Enhancement of Dissolution Rate of Gliclazide Using Solid Dispersions with Polyethylene Glycol 6000. AAPS PharmSciTech, 9, 563-570.

Costa, P., Lobo, J.M.S. 2001. Modeling and comparison of dissolution profiles. Eur. J. Pharm. Sci. 13, 123-133.

Dimitrova, E., Bogdanova, S., Mitcheva, M., Tanev, I. and Minkov. E. 2000. Development of model aqueous ophthalmic solution of indomethacin. Drug Dev. Ind. Pharm. 26, 12971301.

EMFA, 2001. European Agency for the Evaluation of Medicinal Products (EMEA), Notes for Guidance on the Investigation of Bioavailability and Bioequivalence. Available at http://www. emea.europa.eu/pdfs/human. /ewp/140198en.pdf.

FDA. 1997. US Food and Drug Administration, Center for Drug Evaluation and Research, Guidance for industry: Dissolution testing of immediate release solid oral dosage forms (http://www.fda.Gov/cder/guidance/1713bp1.pdf)

Harrower, A.D. 1994. Comparison of efficacy, secondary failure rate and complications of sulfonylurea. J. Diabetes Complicat. 8, 201-203.

Higuchi, T. 1961. Rate of release of medicaments from ointment bases containing drugs in suspension. J. Pharm. Sci. 50, 847875 .

Hixon, A.W. and Crowell, J.H. 1931. Dependence of reaction velocity upon surface and agitation. Ind. Eng. Chem. 23, 923931

Najmuddin, N., Khan, T., Mohsin, A.A. and Patel, V. 2010. Enhancement of dissolution rate of Ketoconazole by solid dispersion. Int. J. Pharm. Pharm. Sci. 2, 132-136.

Palmer,K.J. and Brogden, R.N. 1993. Gliclazide, an update of its pharmacological properties and therapeutic efficacy in NIDDM. Drugs 46, 92-125.

Sekiguchi, K., Obi, N. 1961. Studies on absorption of eutectic mixture. IA comparison of the behavior of eutectic mixture of sulfathiazole and that of ordinary sulfathizaole in man. Chem. Pharm. Bull. 9, 866-872.

Shin, S. C. and. Cho, C. W. 1997. Physicochemical characterizations of piroxicamYpoloxamer solid dispersion. Pharm. Dev. Technol. 2,403Y407.

Tanaka, N., Imai, K., Okimoto, K., Jeda, S.R., Ibuki, Y.T., Higaki, K. Kimura, T. 2006. Developments of novel sustain release system disintegration controlled matrix tablet (DCMT) with solid dispersion granules of nicladipine (II): In vivo evaluation. J. Control. Rel. 122, 51-56.

Wagner. J.G. 1969. Interpretation of present dissolved-time plots derived from in vitro testing of conventional tablets and capsules. J. Pharm. Sci. 58, 1253-1257. 